\title{
The Abscopal Effect: Could a Phenomenon Described Decades Ago Become Key to Enhancing the Response to Immune Therapies in Breast Cancer?
}

\author{
Hans-Christian Kolberg ${ }^{a}$ Oliver Hoffmann ${ }^{b}$ René Baumann ${ }^{c}$ \\ ${ }^{a}$ Department of Gynecology and Obstetrics, Marienhospital Bottrop, Bottrop, Germany; ${ }^{b}$ Department of \\ Gynecology and Obstetrics, University Hospital Essen, Essen, Germany; ${ }^{c}$ Department of Radiation Oncology, \\ St. Marien-Hospital, Siegen, Germany
}

\section{Keywords}

Abscopal effect · Breast cancer · Immune therapy · Radiotherapy

\begin{abstract}
Background: The term "abscopal effect" was defined in 1953. In oncology the term is used to describe systemic antitumor effects triggered by local irradiation (nontarget effect). Although the mechanism of the abscopal effect is not completely understood yet, it has been demonstrated that in situ tumor vaccination, and the resulting antitumor immune response, is one of the key factors. Summary: The development of immune therapies has recently led to concepts combining local radiotherapy and immune therapy with the aim of enhancing the response to immune therapy by the immunological mechanisms summarized in the term abscopal effect. This concept has also been investigated in less immunogenic tumors such as breast cancer. Initial data are promising but the hypothesis that the combination of checkpoint inhibitors and local radiotherapy could be an effective combination in breast cancer has to be proven by ongoing trials. Substitution of local radiotherapy by local hyperthermia could be an option in selected cases. Key Messages: Combination of checkpoint inhibitors with local radiation or hyperthermia in breast cancer is a promising approach and could enhance the response rates generated by immune therapy alone through the antitumor immune response initiated by the abscopal effect.

(c) 2020 S. Karger AG, Basel
\end{abstract}

\section{Introduction}

In 1896 the first patients were treated with $\mathrm{x}$-ray radiotherapy [1], and only 2 decades later, in 1916, the hypothesis that radiation therapy could trigger a systemic effect was discussed. This systemic effect was described by the term radiovaccination [2]. The term "abscopal effect" for this phenomenon was first used in the year 1953 and it was described as "an action at a distance from the irradiated volume but within the same organism" [3]. Whereas this definition referred to any biological effect, in modern oncology the term abscopal effect is used to describe systemic tumor-specific immunity or immunogenic cell death triggered by local therapy.

Another nontarget effect is the radiation-induced bystander effect. This term describes a biological alteration manifested in unirradiated cells [4]. The abscopal effect, though defined almost 70 years ago, has been reported only in rare case reports and mostly in cancer considered to be immunogenic even without intervention, such as renal cell carcinoma, hepatocellular carcinoma, and melanoma [5]. After the implementation of immune checkpoint inhibition as an oncological principle, the abscopal effect became relevant also for tumors considered to be less immunogenic, such as breast cancer, because combining local radiotherapy with immune therapy could result in increased response rates to checkpoint inhibitors. However, enhancing the effects of checkpoint inhibitors by local radiotherapy is not trivial and several caveats 
need to be addressed. Defining optimal radiation doses and timing, choosing the optimal immune therapy agents, and solving the problem of immunosuppressive effects of radiotherapy are still unmet medical needs [6].

In this review, we describe the biological mechanisms and clinical impact of the abscopal effect, discuss combination strategies with immune therapy in breast cancer and abscopal effects triggered by other local therapies such as hyperthermia, and, finally, propose future directions for research aimed at enhancing the response to immune therapies by the abscopal effect in breast cancer.

\section{Abscopal Effect}

The local effects of ionizing radiation on solid malignant tumors were for decades the main explanation for the response of cancer to radiotherapy [7]. These local effects are represented by a cascade initiated by DNA damage leading to apoptosis and autophagy $[8,9]$. From a historical perspective, the systemic effect attributed to radiotherapy was an immunosuppression by induction of a leukopenia through a local cytotoxic impact of radiation on radiated parts of the bone marrow [10]. This immunosuppression was generally attributed to older protocols with large parts of bone marrow as well as the circulating blood volume within the radiation fields [11]. The first signals reporting an influence of the immune system and specifically $\mathrm{T}$ cells on the response to radiotherapy were described in fibrosarcoma and melanoma mouse models. Immunocompetent mice showed a superior response to irradiation compared to T-cell-deficient or T-cell-depleted mice locally, and in the case of the fibrosarcoma model also systemically, by a decreased rate of metastases [12, 13]. A direct stimulation of chemokines with an impact on immune responses through attraction of effector $\mathrm{T}$ cells such as CXCL10 and CXCL16 has also been reported in preclinical breast cancer models [14]. In an in vivo mouse model a breast cancer cell line (67NR) was implanted on 2 sides (both flanks) and 1 location was irradiated with 2 or 6 Gy. Radiotherapy only led to a growth delay in the irradiated tumor, but the combination of radiotherapy and a dendritic cell growth factor (Flt3-L) led to shrinking of the nonirradiated tumor. The authors concluded that the abscopal effect by radiation is immune mediated and requires a T-cell response [15].

Clinical observations of the abscopal effect have been reported in various tumors including melanoma [16], malignant lymphoma [17], hepatocellular carcinoma [5], and kidney cancer [18]. In the case of a metastatic melanoma, ultra-hypofractionated stereotactic body radiotherapy (SBRT) was administrated in 3 fractions to 28.5 Gy in 7 days at a paraspinal mass during progressive disease, combined with a CTLA- 4 antibody. Not only did the irradiated lesion show a good response, but there was also a disease response outside the radiation field with a decrease in lymph node and splenic metastases [16]. In a case report, a patient with Kaposi sarcoma treated with azathioprine because of a previous kidney transplantation responded less to radiation than comparable patients without immunosuppressive therapy [19].

There have also been reports of clinical cases of metastatic breast cancer with beneficial effects on unirradiated lung [20] and bone metastases [21]. It has even been hypothesized that the effect of adjuvant radiotherapy in the curative setting could be an indicator of systemic (i.e., abscopal) effects of local radiation, because the delay in the separation of survival curves in adjuvant radiotherapy trials compared to adjuvant systemic therapy trials is similar to the delay of curves in the majority of randomized immunotherapy trials. However, the number of reported cases with a clear abscopal effect in breast cancer is rare [22].

Although the mechanisms of the abscopal effect are not yet completely understood, the interaction of local radiotherapy and the immune system has been thoroughly investigated. Local tissue destruction by ionizing radiation results in immunogenic cell death, a process that is defined by the release of immunogenic factors summarized by the term damage-associated molecular patterns (DAMP). DAMP include calreticulin, high-mobility group box 1 protein, and adenosine triphosphate. These factors lead to an improved antigen presentation to T cells by activation of dendritic cells. One of the key mechanisms in this process is translocation of calreticulin to the cell surface $[23,24]$. Radiation-induced signals have shown a powerful synergy between radiotherapy and immune agents in experimental settings. There is a growing consensus that immunotherapies and immunomodulation could increase the abscopal effect. A recent systemic review about the combination of radiotherapy and ipilimumab in metastatic melanoma patients reported a median abscopal effect in $26 \%$ of the patients in 16 trials. The patients who experienced an abscopal effect also had a better overall survival [25]. In an ideal scenario radiation should increase immune stimulation and reduce immune suppression, resulting in an enhanced T-cell-mediated killing of tumor cells [26].

If the immune checkpoint receptor programmed cell death 1 (PD-1) is bound to its ligands, i.e., programmed cell death ligands 1 and 2 (PD-L1 and PD-L2), on a CD8 ${ }^{+}$ $\mathrm{T}$ cell, the $\mathrm{T}$-cell response is inhibited. One of the immune suppressive effects of radiotherapy is mediated by PD-L1. PD-L1 expression in tumor cells is activated by radiotherapy, leading to an increased amount of bound PD-1 and therefore reducing the T-cell-mediated killing of tumor cells, an effect that could compromise the long-term prognosis [27]. An important question under investiga- 


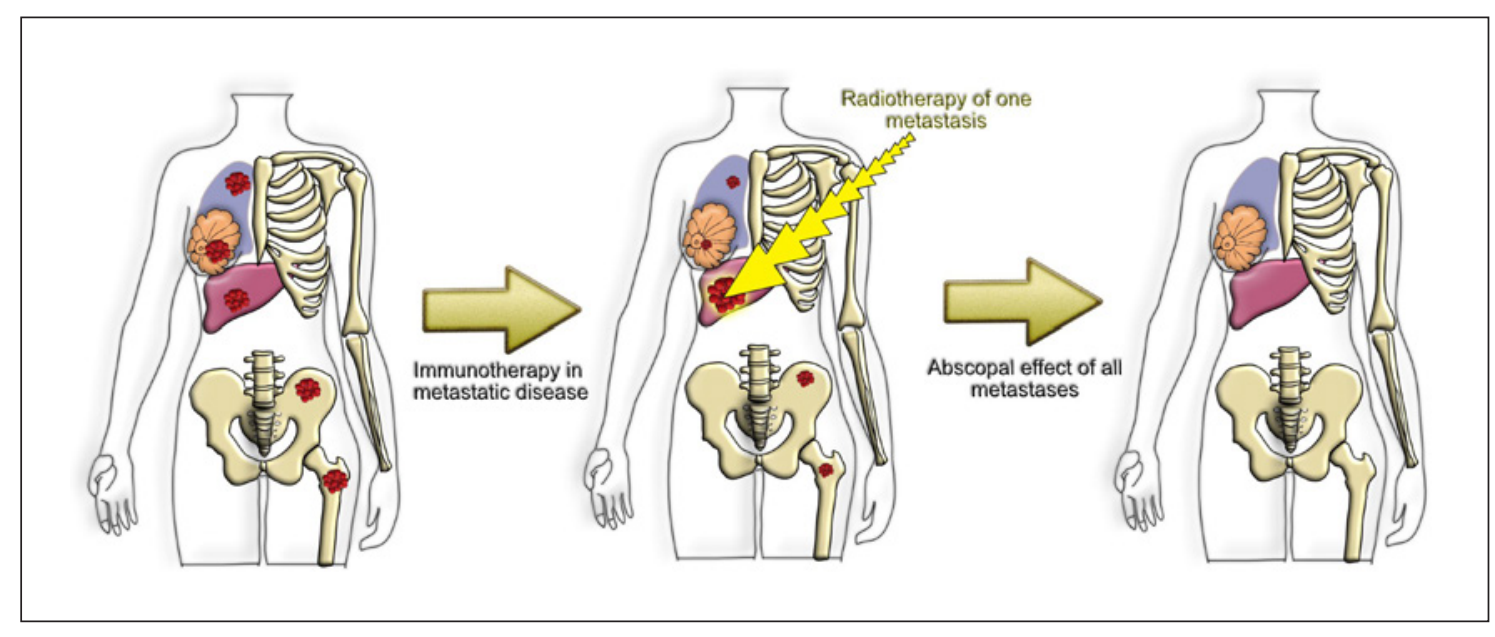

Fig. 1. Combination of immune therapy and radiotherapy.

tion is the radiation dose per fraction needed to trigger an immunogenic effect. It has been demonstrated that irradiation with single doses above 10 Gy leads to an increased Trex 1 amplification and a decrease in interferon- $\beta$ expression. This has a negative effect on the priming of $\mathrm{CD}^{+} \mathrm{T}$ cells [28]. This has led to the proposal that in clinical trials stereotactic radiation (SBRT) doses in combination with immunotherapy should be limited to 6-8 Gy per fraction. The controversial effects of radiotherapy on the immune system, of which we have only mentioned a few key elements here, have led to the concept of enhancing the immunostimulatory part of this equilibrium by immune checkpoint inhibitors and other immune agents aimed at potentiating the in situ vaccination initiated by local radiation [29]. This synergistic approach could play a key role in solving an important task in modern oncology, i.e., the conversion of nonresponders to immune therapy to responders [30], as illustrated in Figure 1.

\section{Immune Therapies and Radiotherapy in Breast Cancer}

The facts that breast cancer is less immunogenic than other tumors and that even in more immunogenic tumors the occurrence rate of a clinically meaningful abscopal effects is low have led to concepts combining local radiation and immune therapy aimed at an enhanced immune antitumor response by the synergistic effects of both strategies [31].

In mouse models the combination of PD-1 or PD-L1 inhibitors with radiotherapy resulted in an improved local control, a longer tumor growth delay, and a better long-term survival $[26,27,32]$.
Clinical trial data supporting this concept are mainly available for melanoma in combination with the PD-1 inhibitor pembrolizumab $[33,34]$ and the CTLA-4 inhibitor ipilimumab [35-37].

Numerous clinical trials have investigated different approaches in breast cancer, but results have only been reported for a small number of these trials to date. In a phase 2 trial the CTLA- 4 inhibitor tremelimumab was combined with brain irradiation in breast cancer patients with brain metastases (NCT02563925). In 2 phase 1 trials the PD-1 inhibitor pembrolizumab was combined with hypofractionated or stereotactic radiotherapy in metastatic breast cancer (NCT02303990 and NCT02303366). The PD-L1 inhibitor durvalumab was combined with the CTLA-4 inhibitor tremelimumab and with 3 fractions of 8 Gy versus 1 fraction of 17 Gy local radiotherapy in metastatic breast cancer in a phase 1 trial (NCT02639026). Nivolumab, a PD-1 inhibitor, was given with or without prior induction therapy (radiotherapy vs. different chemotherapy agents) in metastatic triple-negative breast cancer in a phase 2 trial (NCT02499367). These trials have completed recruitment but the results are still pending.

The combination of local radiation and application of fresolimumab, an antibody neutralizing all isoforms of transforming growth factor (TGF) - $\beta$, in 2 doses ( 1 and 10 $\mathrm{mg} / \mathrm{kg}$ ) in a small trial with 23 patients with metastatic breast cancer demonstrated a better overall survival for the group with radiation and the higher dose of TGF- $\beta$ blockade [38].

A single-arm trial in heavily pretreated patients with metastatic breast cancer with a median of 3 of previous therapies, a combination of local radiotherapy with a dose of $30 \mathrm{~Gy}$ was delivered in 5 daily fractions and pembrolizumab was delivered 2-7 days before radiation and it re- 
Table 1. Ongoing trials combining pembrolizumab and local radiotherapy in breast cancer

\begin{tabular}{|c|c|c|c|c|}
\hline Investigational therapy & Population & Leading site & Status & Clinicaltrials.gov ID \\
\hline $\begin{array}{l}\text { Pembrolizumab and tumor boost radiation } \\
\text { before planned breast surgery or } \\
\text { chemotherapy }\end{array}$ & $\begin{array}{l}\text { Cohort 1: high-risk, ER-positive, and } \\
\text { HER2-negative operable breast cancer } \\
\text { Cohort 2: triple-negative operable breast } \\
\text { cancer }\end{array}$ & $\begin{array}{l}\text { Cedars-Sinai Medical Center, } \\
\text { Los Angeles, CA, USA }\end{array}$ & $\begin{array}{l}\text { Single-arm phase } 1 / 2 \\
\text { recruiting }\end{array}$ & NCT03366844 \\
\hline $\begin{array}{l}\text { Pembrolizumab preoperatively and } \\
\text { intraoperative radiotherapy }\end{array}$ & Triple-negative breast cancer & $\begin{array}{l}\text { Columbia University Medical Center, } \\
\text { New York, NY, USA }\end{array}$ & $\begin{array}{l}\text { Single-arm phase } 1 \\
\text { recruiting }\end{array}$ & NCT02977468 \\
\hline $\begin{array}{l}\text { In situ vaccination with Flt3L, radiation, and } \\
\text { poly-ICLC combined with pembrolizumab }\end{array}$ & $\begin{array}{l}\text { Non-Hodgkin lymphoma, metastatic } \\
\text { breast cancer, and head and neck squamous } \\
\text { cell carcinoma }\end{array}$ & $\begin{array}{l}\text { Icahn School of Medicine at Mount } \\
\text { Sinai, New York, NY, USA }\end{array}$ & $\begin{array}{l}\text { Single-arm phase } 1 / 2 \\
\text { recruiting }\end{array}$ & NCT03789097 \\
\hline $\begin{array}{l}\text { Stereotactic body radiation therapy and } \\
\text { In situ oncolytic virus therapy followed by } \\
\text { pembrolizumab }\end{array}$ & $\begin{array}{l}\text { Metastatic triple-negative breast cancer } \\
\text { and metastatic non-small cell lung cancer }\end{array}$ & $\begin{array}{l}\text { Houston Methodist Cancer Center, } \\
\text { Houston, TX, USA }\end{array}$ & $\begin{array}{l}\text { Single-arm phase } 2 \\
\text { recruiting }\end{array}$ & NCT03004183 \\
\hline $\begin{array}{l}\text { Pembrolizumab with stereotactic body } \\
\text { radiotherapy }\end{array}$ & $\begin{array}{l}\text { Advanced solid tumors (including breast } \\
\text { cancer) }\end{array}$ & $\begin{array}{l}\text { University of Chicago, Chicago, IL, } \\
\text { USA }\end{array}$ & $\begin{array}{l}\text { Single-arm phase } 1 \\
\text { recruiting }\end{array}$ & NCT02608385 \\
\hline $\begin{array}{l}\text { Pembrolizumab + radiotherapy vs. } \\
\text { FLT3L (CDX-301) + radiotherapy }\end{array}$ & $\begin{array}{l}\text { Clinical stage 2-3, HR-positive } \\
\text { HER2-negative breast cancer }\end{array}$ & $\begin{array}{l}\text { Weill Cornell Medicine New York } \\
\text { Presbyterian Hospital, New York, NY, } \\
\text { USA }\end{array}$ & $\begin{array}{l}\text { Randomized phase } 2 \\
\text { recruiting }\end{array}$ & NCT03804944 \\
\hline
\end{tabular}

sulted in durable responses of $33 \%$ outside the radiated field [39], irrespectively of the PD-L1 status of the tumor. In this heavily pretreated population this has to be interpreted as a promising signal, although the low number of patients included in that trial $(n=17)$ warrants caution in the interpretation of the results. In a phase 2 trial, with only 8 patients also in the heavily pretreated setting, investigating the combination of pembrolizumab and radiotherapy delivered to a previously unirradiated field in 5 fractions of $4 \mathrm{~Gy}$ delivered 3 days before radiation, no objective response was observed; the trial was closed early [40]. Although it is very difficult to compare those 2 trials, the different timing and dosing of radiotherapy may have played a role in the discordant results, maybe indicating that a higher dose per fraction could be beneficial. Further data are urgently needed regarding robust benefits for patients treated with combinations of checkpoint inhibitors and radiotherapy and the optimal dosing and timing of local radiotherapy and predictive biomarkers.

Ongoing clinical trials combining pembrolizumab and radiotherapy in early and advanced breast cancer are listed in Table 1.

\section{Immunogenic Effects of High-Intensity Focused Ultrasound and Hyperthermia}

Local approaches of applying heat to tumor tissue have been reported to either initiate an abscopal effect or enhance the abscopal effect of radiotherapy in breast cancer. Treatment with high-intensity focused ultrasound before mastectomy significantly increased the numbers of dendritic cells, macrophages, and $\mathrm{CD}^{+}, \mathrm{CD}^{+}$, and $\mathrm{CD} 8^{+}$ lymphocytes at the margins of treated tumors compared to untreated tumors [41]. On the serum level an increase in the immune related cytokines interleukin (IL)- 6 and IL-10 could be demonstrated after thermal ablation of solid tumors [42]. Immunosuppressive cytokines were significantly reduced after high-intensity focused ultrasound of solid tumors, indicating an immunostimulatory effect of the treatment [43]. These and other observations have led to the consideration that high-intensity focused ultrasound may also induce a reaction similar to the abscopal effect of radiotherapy via, in addition to the destruction of the tumor mass, the release of tumor antigens, the release of damage-associated molecular patterns, and the creation of an in situ antigen depot containing all types of tumor proteins. However, it is generally considered unlikely that this approach alone could generate a consistent protective antitumor immunity. Combinations with immune therapies may boost the immune effects of high-intensity focused ultrasound and thus may lead to a therapeutic approach [44].

In a rat model magnetic seed-mediated hyperthermia led to regression of a nontreated lesion and a significant increase in $\mathrm{CD}^{+}$cells [45]. Other experiments including different tumors and treatment with radiation and magnetic seed-mediated hyperthermia with or without the application of virus-like-nanoparticles showed local and distant tumor responses with a significantly better response than expected by radiation alone, suggesting an effective immune response [46]. 
In a trial investigating whether magnetic iron oxide nanoparticle hyperthermia (MION-HT) can enhance abscopal effects with radiotherapy and immune checkpoint inhibitors in a triple-negative breast cancer mouse model, the combination of local irradiation, MION-HT, and PD-1 or CTLA-4 inhibitors led to a decrease in the local tumor burden and increased $\mathrm{CD}^{+} \mathrm{T}$ lymphocytes. The distant effect of a reduction of lung metastases was modest but measurable [47].

These results indicate that the combination of immune therapies and local hyperthermia might also be a promising approach regarding enhancement of the efficacy of checkpoint inhibitors by the abscopal effect.

\section{Future Directions}

Whereas it has been proven that local radiotherapy can initiate an adaptive antitumor immune response by enhancing the T-cell response through an increased tumor antigen presentation, abscopal effects following radiotherapy with a clinical impact on the course of the disease remain relatively rare. It seems unlikely that this immune response alone is sufficient to produce a clinically meaningful effect on the resistance of malignancies to the immune system. Especially in tumors with a lower immunogenicity, such as breast cancer, the combination of local radiation and systemic therapy with immunomodulatory agents can be a possible solution to low response rates to immune therapies, especially in the metastatic setting, by boosting the immune response by in situ vaccination through the abscopal effect. However, research should not be limited to metastatic disease; approaches to address early stages appear promising, because the lower rate of tumor heterogeneity in these cases may lead to a more effective elimination of residual disease via combined radiotherapy and immune therapy. Especially preoperative studies with intact primary tumors seem to be an optimal setting for the identification of biomarkers indicating a response or resistance to combined radiotherapy and immune therapy [48]. The approach of window-of-opportunity studies in breast cancer with the possibility of shortterm rebiopsies qualifies this disease as a good candidate for further research. The focus of future investigations has to be on the dosing and timing of irradiation in the course of systemic immune therapy and the search for biomarkers indicating the patient groups benefitting from this approach. Furthermore, the substitution of radiotherapy by local hyperthermia in combination concepts with immune checkpoint inhibitors in future studies could lead to the possibility of benefitting from in situ vaccination where ionizing radiation is not indicated or not available.

Another unsolved problem is the heterogeneity between primary tumor and metastases. The discordance regarding PD-1 and PD-L1 expression between breast tumors and their distant metastases has been reported to be as high as 50\% [49] and this phenomenon is present also as inter- and intrametastatic heterogeneity [48]. The question of whether metastases with different immune profiles can be influenced by an immune response generated from local treatment of a primary tumor with a potentially completely discordant immune profile remains unanswered and should be further investigated.

\section{Conclusion}

The abscopal effect of radiotherapy is a phenomenon that was described almost 70 years ago and, in the past, in oncology of solid tumors it was mainly attributed to immunogenic tumors such as melanoma. The development of immune therapies has recently led to concepts combining local radiotherapy and immune therapy with the aim of enhancing the response to immune therapy by the immunological mechanisms summarized in the term abscopal effect. This concept has also been investigated in less immunogenic tumors such as breast cancer. Initial data are promising and, although it is too early for a final evaluation, the combination of checkpoint inhibitors and local radiotherapy or local hyperthermia seems promising in breast cancer. Further randomized clinical trials are urgently needed.

\section{Conflict of Interest Statement}

H.-C.K. has received honoraria from Carl Zeiss meditec, Teva, Theraclion, Novartis, Amgen, AstraZeneca, Pfizer, Janssen-Cilag, GSK, LIV Pharma, Genomic Health, MSD, and Daiichi Sankyo and owns stock in Theraclion and Phaon scientific. O.H. has received honoraria from Roche, Pfizer, Novartis, MSD, Riemser, Daichii Sankyo, and Astra Zeneca. R.B. has received honoraria from Merck, Bristol Myers Squibb, MSD, and Astra Zeneca and travel cost reimbursement from Merck and Varian.

\section{Funding Sources}

The authors declare that they received no funding of any source for the preparation or writing of this work.

\section{Author Contributions}

H.-C.K., O.H., and R.B. substantially contributed to the conception of the paper, drafted or critically revised this work for important intellectual content, and approved the final version to be published. Furthermore, they all agree to be accountable for all aspects of this work in ensuring that questions related to the accuracy or integrity of any part of this work are appropriately investigated and resolved. 


\section{References}

1 Schiff E. The Therapeutical Value of the X Rays in Medicine. BMJ. 1900 May;1(2053): 1082-3.

2 Shohan J. Some Theoretical Considerations on the Present Status of Roentgen Therapy. Boston Med Surg J. 1916 Sep;175(10):321-7.

3 Mole RH. Whole body irradiation; radiobiology or medicine? Br J Radiol. 1953 May; 26(305):234-41.

4 Kaminski JM, Shinohara E, Summers JB, Niermann KJ, Morimoto A, Brousal J. The controversial abscopal effect. Cancer Treat Rev. 2005 May;31(3):159-72.

5 Reynders K, Illidge T, Siva S, Chang JY, De Ruysscher D. The abscopal effect of local radiotherapy: using immunotherapy to make a rare event clinically relevant. Cancer Treat Rev. 2015 Jun;41(6):503-10.

$6 \mathrm{Hu}$ ZI, McArthur HL, Ho AY. The Abscopal Effect of Radiation Therapy: What Is It and How Can We Use It in Breast Cancer? Curr Breast Cancer Rep. 2017;9(1):45-51.

7 Ross GM. Induction of cell death by radiotherapy. Endocr Relat Cancer. 1999 Mar;6(1): 41-4

8 Dewey WC, Ling CC, Meyn RE. Radiationinduced apoptosis: relevance to radiotherapy. Int J Radiat Oncol Biol Phys. 1995 Nov;33(4): 781-96.

9 Eriksson D, Stigbrand T. Radiation-induced cell death mechanisms. Tumour Biol. 2010 Aug;31(4):363-72.

10 Blomgren H, Glas U, Melén B, Wasserman J. Blood lymphocytes after radiation therapy of mammary carcinoma. Acta Radiol Ther Phys Biol. 1974 Jun;13(3):185-200.

11 Grilli G, Nothdurft W, Fliedner TM. Radiation sensitivity of human erythropoietic and granulopoietic progenitor cells in the blood and in the bone marrow. Int J Radiat Biol Relat Stud Phys Chem Med. 1982 Jun;41(6): 685-7.

12 Stone HB, Peters LJ, Milas L. Effect of host immune capability on radiocurability and subsequent transplantability of a murine fibrosarcoma. J Natl Cancer Inst. 1979 Nov; 63(5):1229-35.

13 Lee Y, Auh SL, Wang Y, Burnette B, Wang Y, Meng Y, et al. Therapeutic effects of ablative radiation on local tumor require $\mathrm{CD} 8+\mathrm{T}$ cells: changing strategies for cancer treatment. Blood. 2009 Jul;114(3):589-95.

14 Matsumura S, Wang B, Kawashima N, Braunstein S, Badura M, Cameron TO, et al. Radiation-induced CXCL16 release by breast cancer cells attracts effector T cells. J Immunol. 2008 Sep;181(5):3099-107.

15 Demaria S, Ng B, Devitt ML, Babb JS, Kawashima $\mathrm{N}$, Liebes $\mathrm{L}$, et al. Ionizing radiation inhibition of distant untreated tumors $(\mathrm{ab}$ scopal effect) is immune mediated. Int J Radiat Oncol Biol Phys. 2004 Mar;58(3):862-70.

16 Postow MA, Callahan MK, Barker CA, Yamada Y, Yuan J, Kitano S, et al. Immunologic correlates of the abscopal effect in a patient with melanoma. N Engl J Med. 2012 Mar; 366(10):925-31.
17 Antoniades J, Brady LW, Lightfoot DA. Lymphangiographic demonstration of the abscopal effect in patients with malignant lymphomas. Int J Radiat Oncol Biol Phys. 1977 JanFeb;2(1-2):141-7.

18 Robin HI, AuBuchon J, Varanasi VR, Weinstein $\mathrm{AB}$. The abscopal effect: demonstration in lymphomatous involvement of kidneys. Med Pediatr Oncol. 1981;9(5):473-6.

19 Holecek MJ, Harwood AR. Radiotherapy of Kaposi's sarcoma. Cancer. 1978 May;41(5): 1733-8.

20 Azami A, Suzuki N, Azami Y, Seto I, Sato A, Takano Y, et al. Abscopal effect following radiation monotherapy in breast cancer: A case report. Mol Clin Oncol. 2018 Sep;9(3):283-6.

21 Leung HW, Wang SY, Jin-Jhih H, Chan AL. Abscopal effect of radiation on bone metastases of breast cancer: A case report. Cancer Biol Ther. 2018 Jan;19(1):20-4.

22 Jatoi I, Benson JR, Kunkler I. Hypothesis: can the abscopal effect explain the impact of adjuvant radiotherapy on breast cancer mortality? NPJ Breast Cancer. 2018 Apr;4:8.

23 Barker HE, Paget JT, Khan AA, Harrington KJ. The tumour microenvironment after radiotherapy: mechanisms of resistance and recurrence. Nat Rev Cancer. 2015 Jul;15(7): 409-25.

24 Gameiro SR, Jammeh ML, Wattenberg MM, Tsang KY, Ferrone S, Hodge JW. Radiationinduced immunogenic modulation of tumor enhances antigen processing and calreticulin exposure, resulting in enhanced T-cell killing. Oncotarget. 2014 Jan;5(2):403-16.

25 Chicas-Sett R, Morales-Orue I, RodriguezAbreu D, Lara-Jimenez P. Combining radiotherapy and ipilimumab induces clinically relevant radiation-induced abscopal effects in metastatic melanoma patients: A systematic review. Clin Transl Radiat Oncol. 2017 Dec;9: $5-11$.

26 Wu CT, Chen WC, Chang YH, Lin WY, Chen MF. The role of PD-L1 in the radiation response and clinical outcome for bladder cancer. Sci Rep. 2016 Jan;6(1):19740.

27 Sharabi AB, Tran PT, Lim M, Drake CG, Deweese TL. Stereotactic radiation therapy combined with immunotherapy: augmenting the role of radiation in local and systemic treatment. Oncology (Williston Park). 2015 May; 29(5):331-40.

28 Vanpouille-Box C, Alard A, Aryankalayil MJ, Sarfraz Y, Diamond JM, Schneider RJ, et al. DNA exonuclease Trexl regulates radiotherapy-induced tumour immunogenicity. Nat Commun. 2017 Jun;8(1):15618.

29 Liu Y, Dong Y, Kong L, Shi F, Zhu H, Yu J. Abscopal effect of radiotherapy combined with immune checkpoint inhibitors. J Hematol Oncol. 2018 Aug;11(1):104.

30 Tolba MF, Omar HA. Immunotherapy, an evolving approach for the management of triple negative breast cancer: converting nonresponders to responders. Crit Rev Oncol Hematol. 2018 Feb;122:202-7.

31 Vatner RE, Cooper BT, Vanpouille-Box C, Demaria S, Formenti SC. Combinations of immunotherapy and radiation in cancer therapy. Front Oncol. 2014 Nov;4:325
32 Liang H, Deng L, Chmura S, Burnette B, Liadis N, Darga T, et al. Radiation-induced equilibrium is a balance between tumor cell proliferation and $\mathrm{T}$ cell-mediated killing. J Immunol. 2013 Jun;190(11):5874-81.

33 Roger A, Finet A, Boru B, Beauchet A, Mazeron JJ, Otzmeguine Y, et al. Efficacy of combined hypo-fractionated radiotherapy and anti-PD-1 monotherapy in difficult-to-treat advanced melanoma patients. OncoImmunology. 2018 Mar;7(7):e1442166.

34 Aboudaram A, Modesto A, Chaltiel L, Gomez-Roca C, Boulinguez S, Sibaud V, et al. Concurrent radiotherapy for patients with metastatic melanoma and receiving anti-programmed-death 1 therapy: a safe and effective combination. Melanoma Res. 2017 Oct;27(5): 485-91.

35 Theurich S, Rothschild SI, Hoffmann M, Fabri M, Sommer A, Garcia-Marquez $\mathrm{M}$, et al. Local tumor treatment in combination with systemic ipilimumab immunotherapy prolongs overall survival in patients with advanced malignant melanoma. Cancer Immunol Res. 2016 Sep;4(9):744-54.

36 Koller KM, Mackley HB, Liu J, Wagner H, Talamo G, Schell TD, et al. Improved survival and complete response rates in patients with advanced melanoma treated with concurrent ipilimumab and radiotherapy versus ipilimumab alone. Cancer Biol Ther. 2017 Jan; 18(1):36-42.

37 Twyman-Saint Victor C, Rech AJ, Maity A Rengan R, Pauken KE, Stelekati E, et al. Radiation and dual checkpoint blockade activate non-redundant immune mechanisms in cancer. Nature. 2015 Apr;520(7547):373-7.

38 Formenti SC, Lee P, Adams S, Goldberg JD, Li X, Xie MW, et al. Focal irradiation and systemic TGFbeta blockade in metastatic breast cancer. Clin Cancer Res. 2018 Jun;24(11) 2493-504.

39 Ho AY, Barker CA, Arnold BB, Powell SN, Hu ZI, Gucalp A, et al. A phase 2 clinical trial assessing the efficacy and safety of pembrolizumab and radiotherapy in patients with metastatic triple-negative breast cancer. Cancer. 2020 Feb;126(4):850-60.

40 Barroso-Sousa R, Krop IE, Trippa L, TanWasielewski Z, Li T, Osmani W, et al. A Phase II Study of Pembrolizumab in Combination With Palliative Radiotherapy for Hormone Receptor-positive Metastatic Breast Cancer. Clin Breast Cancer. 2020 Jun;20(3):238-45.

41 Xu ZL, Zhu XQ, Lu P, Zhou Q, Zhang J, Wu F. Activation of tumor-infiltrating antigen presenting cells by high intensity focused ultrasound ablation of human breast cancer. Ultrasound Med Biol. 2009 Jan;35(1):50-7.

42 Erinjeri JP, Thomas CT, Samoilia A, Fleisher M, Gonen M, Sofocleous CT, et al. Imageguided thermal ablation of tumors increases the plasma level of interleukin- 6 and interleukin-10. J Vasc Interv Radiol. 2013 Aug;24(8): 1105-12.

43 Zhou Q, Zhu XQ, Zhang J, Xu ZL, Lu P, Wu F. Changes in circulating immunosuppressive cytokine levels of cancer patients after high intensity focused ultrasound treatment. Ultrasound Med Biol. 2008 Jan;34(1):81-7. 
44 van den Bijgaart RJ, Eikelenboom DC, Hoogenboom M, Fütterer JJ, den Brok MH, Adema GJ. Thermal and mechanical high-intensity focused ultrasound: perspectives on tumor ablation, immune effects and combination strategies. Cancer Immunol Immunother. 2017 Feb;66(2):247-58.

45 Wang H, Zhang L, Shi Y, Javidiparsijani S, Wang G, Li X, et al. Abscopal antitumor immune effects of magnet-mediated hyperthermia at a high therapeutic temperature on Walker-256 carcinosarcomas in rats. Oncol Lett. 2014 Mar;7(3):764-70.
46 Hoopes PJ, Moodie KL, Petryk AA, PetrykJD, Sechrist S, Gladstone DJ. Hypo-fractionated Radiation, Magnetic Nanoparticle Hyperthermia and a Viral Immunotherapy Treatment of Spontaneous Canine Cancer. Proc SPIE Int Soc Opt Eng. 2017 Jan-Feb;10066: 1006605.

47 Oei AL, Korangath P, Mulka K, Helenius M, Coulter JB, Stewart J, et al. Enhancing the abscopal effect of radiation and immune checkpoint inhibitor therapies with magnetic nanoparticle hyperthermia in a model of metastatic breast cancer. Int J Hyperthermia. 2019 Nov;36 suppl 1:47-63.
48 Formenti SC, Demaria S. Future of Radiation and Immunotherapy. Int J Radiat Oncol Biol Phys. 2020 Sep;108(1):3-5.

49 Manson QF, Schrijver WA, Ter Hoeve ND, Moelans CB, van Diest PJ. Frequent discordance in PD-1 and PD-L1 expression between primary breast tumors and their matched distant metastases. Clin Exp Metastasis. 2019 Feb;36(1):29-37. 